\title{
ON SOME CLASSES OF ALMOST PERIODIC FUNCTIONS IN ABSTRACT SPACES
}

\author{
DARIUSZ BUGAJEWSKI and GASTON M. N'GUÉRÉKATA
}

Received 15 August 2004

\begin{abstract}
We deal with $C^{(n)}$-almost periodic functions taking values in a Banach space. We give several properties of such functions, in particular, we investigate their behavior in view of differentiation as well as integration. The superposition operator acting in the space of such functions is also under consideration. Some applications to ordinary as well as partial differential equations are presented. Moreover, we introduce the class of the so-called asymptotically $C^{(n)}$-almost periodic functions and give some of their properties.
\end{abstract}

2000 Mathematics Subject Classification: 42A75, 34C27.

1. Introduction. $C^{(n)}$-almost periodic functions are one of the most important generalizations of the concept of almost periodic functions in the sense of Bohr. This generalization relies on the requirement that a given function as well as its derivatives up to the $n$th order inclusively are almost periodic in the sense of Bohr. Many properties of such functions with real values are given in [1,2].

In the present paper, we deal with $C^{(n)}$-almost periodic functions taking values in a Banach space. This work is organized in the following way: in Section 2, we give the definition of vector-valued $C^{(n)}$-almost periodic functions and we prove that the linear space of such functions considered with a suitable norm turns out to be a Banach space. Moreover, we give an application to an ordinary linear homogeneous differential equation, where the corresponding operator $A$ on the right side is linear and compact. In Section 3, we investigate the behavior of $C^{(n)}$-almost periodic functions with respect to differentiation as well as integration. Further, we prove that the $C^{(n)}$-almost periodicity of the initial functions $f$ and $g$ imply that the solutions of the classical wave equations in view of the first variable are $C^{(n)}$-almost periodic. Next, in Section 4, we investigate the superposition operator acting in the space of $C^{(n)}$-almost periodic functions. In particular, we give examples of such functions and we consider the case of functions of two variables. Further, in Section 5, we introduce a new class of the so-called asymptotically $C^{(n)}$-almost periodic functions and give several of their properties.

2. Definitions and basic properties. Let $(E,\|\cdot\|)$ be a Banach space. We will use the following notation: $f_{h}(x)=f(h+x)$, where $f: \mathbb{R} \rightarrow E$ and $h, x \in \mathbb{R}$.

Denote by $C^{(n)}(\mathbb{R}, E)$ the linear space of all functions $\mathbb{R} \rightarrow E$ which have a continuous $n$th derivative on $\mathbb{R}$. By $C_{B}^{(n)}(\mathbb{R}, E)$, we will denote the subspace of $C^{(n)}(\mathbb{R}, E)$ 
(briefly: $C^{(n)}(E)$ ) consisting of such functions $f: \mathbb{R} \rightarrow E$ for which

$$
\sup _{t \in \mathbb{R}}\left(\|f(t)\|+\sum_{i=1}^{n}\left\|f^{(i)}(t)\right\|\right)<+\infty
$$

where $f^{(i)}$ denotes the $i$ th derivative of $f$. In the space $C_{B}^{(n)}(\mathbb{R}, E)$ (briefly: $C_{B}^{(n)}(E)$ ) we introduce the norm

$$
\|f\|_{n}=\sup _{t \in \mathbb{R}}\left(\|f(t)\|+\sum_{i=1}^{n}\left\|f^{(i)}(t)\right\|\right) \text { for } f \in C_{B}^{(n)}(E) .
$$

It is well known that $C_{B}^{(n)}(E)$ with this norm is a Banach space.

DeFinition 2.1. A number $\tau \in \mathbb{R}$ is said to be a $\left(\|\cdot\|_{n}, \varepsilon\right)$-almost period (briefly: $\left(\|\cdot\|_{n}, \varepsilon\right)$-a.p.) of a function $f \in C^{(n)}(E)$, if $\left\|f-f_{\tau}\right\|_{n} \leq \varepsilon$ for $\varepsilon>0$. The set of all $\left(\|\cdot\|_{n}, \varepsilon\right)$-almost periods of a function $f$ will be denoted by $E^{(n)}(\varepsilon ; f)$.

DEFINITION 2.2. A function $f \in C^{(n)}(\mathbb{R}, E)$ is said to be $C^{(n)}$-almost periodic (briefly: $C^{(n)}$-a.p.), if for every $\varepsilon>0$ the set $E^{(n)}(\varepsilon ; f)$ is relatively dense in $\mathbb{R}$.

It can be proved easily using the continuity of $f^{(i)}, i=1,2, \ldots, n$, that this set of $\left(\|\cdot\|_{n}, \varepsilon\right)$-almost periods is a closed set.

Denote by $A P^{(n)}(E)$ the set of all $C^{(n)}$-a.p. functions.

Directly from the above definitions it follows that $A P^{(n+1)}(E) \subset A P^{(n)}(E)$. Moreover, putting $n=0$, we have $A P^{(0)}(E)=A P(E)$, where $A P(E)$ denotes the classical Banach space of all almost periodic functions $\mathbb{R} \rightarrow E$, in the sense of Bohr.

Now, we prove the following lemma.

LEMMA 2.3. $A P^{(n)}(E) \subset C_{B}^{(n)}(E)$.

Proof. Let $f \in A P^{(n)}(E)$ and let $\varepsilon>0$. For $t \in \mathbb{R}$, we have

$$
\|f(t)\|+\sum_{i=1}^{n}\left\|f^{(i)}(t)\right\| \leq\left\|f(t)-f_{\tau}(t)\right\|+\sum_{i=1}^{n}\left\|f^{(i)}(t)-f_{\tau}^{(i)}(t)\right\|+\left\|f_{\tau}(t)\right\|+\sum_{i=1}^{n}\left\|f_{\tau}^{(i)}(t)\right\| .
$$

Fix $t \in \mathbb{R}$. Since $f \in A P^{(n)}(E)$, there exists $\left(\|\cdot\|_{n}, \varepsilon\right)$-a.p. $\tau=(\varepsilon, t) \in(-t,-t+l)$, where $l=l(\varepsilon)>0$ is a number which characterizes the relative density of $E^{(n)}(\varepsilon ; f)$. Moreover, since $f \in C^{(n)}(E)$, there exists $M>0$ such that

$$
\max _{s \in[0, l]}\left(\|f(s)\|+\sum_{i=1}^{n}\left\|f^{(i)}(s)\right\|\right) \leq M
$$

where $s=t+\tau$. Thus, $\|f\|_{n}<+\infty$, which completes the proof. 
In what follows, we will need the following well-known lemma.

LEMMA 2.4. Let $f, g \in A P(E)$. Then, for every $\varepsilon>0$ there exists a relatively dense set of their common $\varepsilon$-a.p.

An immediate consequence of Lemma 2.4 is the following criterion.

THEOREM 2.5. $f \in A P^{(n)}(E)$ if and only if $f^{(i)} \in A P(E)$ for $i=0,1, \ldots, n$.

By the above result, we infer that if $f \in A P^{(n)}(E)$, then the range of $f^{(i)}$ is relatively compact for each $i=0,1, \ldots, n$ (see [7, Remark 3.1.6, page 53]).

Now, we recall the very important Bochner's criterion.

THEOREM 2.6. A continuous function $f: \mathbb{R} \rightarrow E$ is almost periodic if and only if for every sequence of real numbers $\left(s_{n}^{\prime}\right)$, there exists a subsequence $\left(s_{n}\right)$ such that $\left(f\left(t+s_{n}\right)\right)$ converges uniformly in $t \in \mathbb{R}$.

See, for example, [7, Theorem 3.1.8, page 55].

It is clear that based on Theorem 2.6, we are able to formulate an analogue of Bochner's criterion for the case of $C^{(n)}$-almost periodicity.

THEOREM 2.7. A function $f \in C^{(n)}(E)$ is $C^{(n)}$-almost periodic if and only if for every sequence of real numbers $\left(s_{n}^{\prime}\right)$, there exists a subsequence $\left(s_{n}\right)$ such that $\left(f^{(i)}\left(t+s_{n}\right)\right)$ converges uniformly in $t \in \mathbb{R}$ for $i=0,1, \ldots, n$.

COROLlaRY 2.8. Let $(T(t))_{t \in \mathbb{R}}$ be a $C_{0}$-group of bounded linear operators on $E$ and for an $e \in E$, the function $x_{e}: \mathbb{R} \rightarrow E$ defined by $x_{e}(t)=T(t) e$ satisfies the assumptions:

(1) $x_{e} \in C^{(n)}(E)$ for some $n$;

(2) $R_{x_{e}}$ is relatively compact in $E$, where $R_{x_{e}}$ denotes the range of the function $x_{e}$. Then, $x_{e} \in A P^{(n)}(E)$.

Proof. It is an immediate consequence of Theorem 2.7 and the group property.

Application 2.9. Consider in a Banach space $E$ the abstract differential equation

$$
x^{\prime}(t)=A x(t) \text { for } t \in \mathbb{R},
$$

where $A \in L(E)$ is a compact linear operator. Let $x(t)$ be a solution of the above equation.

Then, the following hold:

(1) if $R_{x}$ is bounded, then $x^{\prime} \in A P^{n}(E)$ for all $n=1,2, \ldots$,

(2) if $R_{x}$ is relatively compact, then $x \in A P^{n}(E)$ for all $n=0,1,2, \ldots$

Proof. Clearly, $x(t)=e^{t A} x(0) \in C^{n}(E)$, for all $n=0,1,2, \ldots$, since $A$ is continuous and defined on all $E$.

To prove assertion (1), we suppose that $x(t)$ is bounded; then $A x(t)$, and consequently, $x^{\prime}(t)$ is relatively compact since $A$ is compact. Thus, for all $n=1,2, \ldots$, the function $x^{\prime}(t)=A e^{t A} x(0)=e^{t A} A x(0)$ is $C^{n}$-almost periodic by Corollary 2.8. 
To prove assertion (2), it suffices to observe that $x(t)=e^{t A} x(0)$; thus obviously $x \in A P^{n}(E)$ for all $n=0,1,2, \ldots$, by Corollary 2.8 .

We have also the following simple proposition.

Proposition 2.10. A linear combination of $C^{(n)}$-a.p. functions is a $C^{(n)}$-a.p. function. Moreover, let $E$ be a Banach space over the field $K(K=\mathbb{R}$ or $\mathbb{C})$. If $f \in A P^{(n)}(E)$ and $v \in A P^{(n)}(K)$, then the following functions are also in $A P^{(n)}(E)$ :

(i) $v f$,

(ii) $f_{a}(t):=f(t+a)$, where $a \in \mathbb{R}$ is fixed,

(iii) $h(t):=f(-t), t \in \mathbb{R}$.

Proof. It is a simple consequence of Theorem 2.7.

THEOREM 2.11. If a sequence $\left(f_{k}\right), k \in \mathbb{N}$, of $C^{(n)}$-a.p. functions is such that $\| f_{k}-$ $f \|_{n} \rightarrow 0$ as $k \rightarrow \infty$, then $f$ is $C^{(n)}$-a.p. function.

Proof. Fix $\varepsilon>0$. There exists $k_{0} \in \mathbb{N}$ such that $\left\|f-f_{k_{0}}\right\|_{n} \leq \varepsilon / 3$. For $\tau \in E^{(n)}\{\varepsilon / 3$; $\left.f_{k_{0}}\right\}$ we obtain

$$
\left\|f-f_{\tau}\right\|_{n} \leq\left\|f-f_{k_{0}}\right\|_{n}+\left\|f_{k_{0}}-\left(f_{k_{0}}\right)_{\tau}\right\|_{n}+\left\|\left(f_{k_{0}}\right)_{\tau}-f_{\tau}\right\|_{n} \leq \varepsilon,
$$

so $E^{(n)}\left(\varepsilon / 3 ; f_{k_{0}}\right) \subset E^{(n)}(\varepsilon ; f)$, and thus $f \in A P^{(n)}(E)$.

COROLLARY 2.12. $A P^{(n)}(E)$ considered with the norm (2.2) turns out to be a Banach space.

Proof. In view of Proposition 2.10 and Lemma 2.3, $A P^{(n)}(E)$ is a linear subspace of $C_{B}^{(n)}(E)$. Let $\left(f_{k}\right), f_{k} \in A P^{(n)}(E), k \in \mathbb{N}$, be a Cauchy sequence. Then, there exists $f \in C_{B}^{(n)}(E)$ such that $\lim _{k \rightarrow \infty}\left\|f_{k}-f\right\|_{n}=0$. By Theorem 2.11, $f \in A P^{(n)}(E)$, so it is a Banach space.

3. Differentiation and integration. The first result in this Section gives a sufficient condition which guarantees that the derivative of a function $f \in A P^{(n)}(E)$ is also a $C^{(n)}$-a.p. function.

THEOREM 3.1. If $f \in A P^{(n)}(E)$ and $f^{(n+1)}$ is uniformly continuous, then $f^{\prime}$ is $C^{(n)}$-a.p. function.

Proof. Fix $\varepsilon>0$. In view of Theorem 2.5 and the uniform continuity of $f^{(n+1)}$, there exists $\delta>0$ such that for $h \in \mathbb{R}$ with $|h|<\delta$ we have

$$
\sum_{i=1}^{n+1}\left\|f^{(i)}(t)-f_{h}^{(i)}(t)\right\| \leq \varepsilon \quad \forall t \in \mathbb{R} .
$$


Further, for $t \in \mathbb{R}$ and $h \neq 0$, we have

$$
\begin{aligned}
& \frac{f(t+h)-f(t)}{h}-f^{\prime}(t)=\frac{1}{h} \int_{0}^{h}\left(f^{\prime}(s+t)-f^{\prime}(t)\right) d s \\
& \left\|\frac{f_{h}-f}{h}-f^{\prime}\right\|_{n} \\
& \quad=\sup _{t \in \mathbb{R}}\left(\left\|\frac{1}{h} \int_{0}^{h}\left(f^{\prime}(s+t)-f^{\prime}(t)\right) d s\right\|+\sum_{i=1}^{n}\left\|\frac{d^{i}}{d t^{i}}\left(\frac{1}{h} \int_{0}^{h}\left(f^{\prime}(s+t)-f^{\prime}(t)\right) d s\right)\right\|\right) \\
& \quad \leq \sup _{t \in \mathbb{R}} \frac{1}{h} \int_{0}^{h}\left(\sum_{i=1}^{n+1}\left\|\frac{d^{i}}{d t^{i}} f(s+t)-f^{(i)}(t)\right\|\right) d s .
\end{aligned}
$$

Thus, by (3.1), we obtain $\left\|\left(f_{h}-f\right) / h-f^{\prime}\right\|_{n} \leq \varepsilon$ for each $h \in \mathbb{R}$ with $|h|<\delta$.

Now, we apply Theorem 2.11 and the proof is complete.

We recall now the well-known Bohl-Bohr-type theorem (see, e.g., [7, Theorem 3.2.6, page 62]).

TheOrem 3.2 (see [7, Theorem 3.2.6, page 62]). Let $f \in A P(E)$ and let the function $F: \mathbb{R} \rightarrow E$ defined by $F(t)=\int_{0}^{t} f(s) d s$; then $F \in A P(E)$ if and only if its range $R_{F}=\{F(t)$ : $t \in \mathbb{R}\}$ is relatively compact in $E$.

One can extend this result in the following way.

THEOREM 3.3. If $f \in A P(E)$ and the range $R_{F}$ is relatively compact, then $F \in$ $A P^{(1)}(E)$.

Proof. In view of Bohl-Bohr theorem, $F \in A P(E)$. Moreover, since $f$ is continuous, $F^{\prime}(t)=f(t)$ for $t \in \mathbb{R}$. Thus, $F^{\prime} \in A P(E)$ and, consequently, $F \in A P^{(1)}(E)$.

Theorem 3.3 is a special case of the following theorem.

THEOREM 3.4. If $f \in A P^{(n)}(E)$ and the range $R_{F}$ is relatively compact, then $F \in$ $A P^{(n+1)}(E)$.

Proof. In view of Bohl-Bohr theorem, $F \in A P(E)$. Moreover, $F^{\prime}=f \in A P^{(n)}(E)$. In view of Theorem 2.5, we get $F \in A P^{(n+1)}(E)$.

COROLLARY 3.5. If $f^{\prime} \in A P^{(n)}(E)$ and the range $R_{f}$ is relatively compact, then $f \in$ $A P^{(n+1)}(E)$.

Proof. Since $f^{\prime}$ is continuous on $\mathbb{R}$, so,

$$
f(t)=f(0)+\int_{0}^{t} f^{\prime}(s) d s \text { for } t \in \mathbb{R} .
$$

In view of Theorem 3.4, $f \in A P^{(n+1)}(E)$.

EXAMPLE 3.6. Let $f \in A P^{(n)}(E)$, and for a fixed $a \in \mathbb{R}$, define a function $F: \mathbb{R} \rightarrow E$ by $F_{a}(t):=\int_{t}^{t+a} f(s) d s$ for each $t \in \mathbb{R}$. 
Then, $F \in A P^{(n)}(E)$. Moreover, $f$ and $F$ have the same $\left(\|\cdot\|_{n}, \varepsilon\right)$-almost periods.

First, we note that by a suitable change of variable we get $F_{a}(t)=\int_{0}^{a} f(t+\xi) d \xi$. It is then easy to check that $F \in C^{(n)}(E)$.

Now, let $\tau \in E^{(n)}(\epsilon /|a|, f)$; then, for any $t \in \mathbb{R}$, we have

$$
\left\|F_{a}(t+\tau)-F_{a}(t)\right\|_{n} \leq \int_{0}^{a}\|f(t+\tau+\xi)-f(t+\xi)\|_{n} d \xi \leq|a| \frac{\epsilon}{|a|}=\epsilon,
$$

that is $\tau \in E^{(n)}(\epsilon /|a|, F)$, which proves our assertion.

EXAMPLE 3.7. Consider the wave equation

$$
u_{x x}(x, t)=u_{t t}(x, t), \quad u(x, 0)=f(x), \quad u_{t}(x, 0)=g(x) .
$$

Assume that both $f, g \in A P^{(n)}(\mathbb{R})$. Then, for each $t_{0} \in \mathbb{R}$, the solution $u\left(x, t_{0}\right) \in A P^{(n)}$. Indeed, we have

$$
u\left(x, t_{0}\right)=\frac{1}{2}\left(f\left(x+t_{0}\right)+f\left(x-t_{0}\right)\right)+\frac{1}{2} \int_{x-t_{0}}^{x+t_{0}} g(s) d s .
$$

Clearly, $(1 / 2)\left(f\left(x+t_{0}\right)+f\left(x-t_{0}\right)\right) \in A P^{(n)}(\mathbb{R})$ by Proposition 2.10. Also, if we write $\int_{x-t_{0}}^{x+t_{0}} g(s) d s=\int_{x-t_{0}}^{0} g(s) d s+\int_{0}^{x+t_{0}} g(s) d s$, we deduce that it is in $A P^{(n)}(\mathbb{R})$ by the previous example.

4. Superposition operator. Let $E_{1}, E_{2}$ be Banach spaces. We begin this section with the following proposition.

Proposition 4.1. If $A: E_{1} \rightarrow E_{2}$ is bounded linear operator and $f \in A P^{(n)}\left(E_{1}\right)$, then Af $\in A P^{(n)}\left(E_{2}\right)$.

Proof. It is clear that $(A f)^{(n)}=A f^{(n)}$. Then, it is enough to apply Theorem 2.5 and [7, Corollary 2.1.6, page 14] for almost periodic functions.

For every $f \in A P^{(n)}\left(E_{1}\right)$, define the $E_{2}$-valued function $G$ as follows:

$$
G(t):=\int_{0}^{t} A f(s) d s
$$

where $A: E_{1} \rightarrow E_{2}$ is a bounded linear operator.

We have the following corollary.

COROLLARY 4.2. Let $A: E_{1} \rightarrow E_{2}$ be a bounded linear operator with relatively compact range, then the $E_{2}$-valued function $G$ defined above is in $A P^{(n+1)}\left(E_{2}\right)$.

Proof. According to Proposition 4.1, $A f \in A P^{(n)}\left(E_{2}\right)$ for every $f \in A P^{(n)}\left(E_{1}\right)$. We also note that $R_{G} \subset R(A)$, where $R(A)$ is the range of the operator $A$, hence $R_{G}$ is relatively compact. We now complete the proof using Theorem 3.4.

In the Corollary 4.2, note that if the operator $A$ is compact (or of finite rank), the stated result holds. 
Now, we will consider the superposition operator (the autonomous case) acting on the space $A P^{(n)}\left(E_{1}\right)$.

We will utilize the fact that the range $R_{f}=\{f(t) \mid t \in \mathbb{R}\}$ of a $C^{(n)}$-a.p. function is relatively compact to prove the following theorem.

THEOREM 4.3. If $\phi \in C^{(n)}\left(E_{1}, E_{2}\right)$ and $f \in A P^{(n)}\left(E_{1}\right)$, then $\phi \circ f \in A P^{(n)}\left(E_{2}\right)$.

Proof. First, we observe that the result holds for $n=0$ (see [7, Theorem 2.1.5, page 14] for almost periodic functions). Now, we show that it holds for $n=1$. We have $(\phi \circ f)^{\prime}(x)=\phi^{\prime}(f(x)) \circ f^{\prime}(x)$ for $x \in E_{1}$. Since $f^{\prime} \in A P\left(E_{1}\right)=A P\left(L\left(\mathbb{R}, E_{1}\right)\right)$ and $\phi^{\prime} \circ f \in A P\left(L\left(E_{1}, E_{2}\right)\right)$, so $(\phi \circ f)^{\prime} \in A P\left(E_{2}\right)=A P\left(L\left(\mathbb{R}, E_{2}\right)\right)$. Suppose the result holds for $n-1$. This implies that $\phi^{\prime} \circ f \in A P^{(n-1)}\left(L\left(E_{1}, E_{2}\right)\right)$. Moreover, $f^{\prime} \in A P^{(n-1)}\left(E_{1}\right)$, so $(\phi \circ f)^{\prime} \in A P^{(n-1)}\left(E_{2}\right)$. Moreover, $R_{\phi \circ f}$ is relatively compact, so, by Theorem 3.4, $\phi \circ f \in A P^{(n)}\left(E_{2}\right)$.

EXAmple 4.4. Having Theorem 4.3, one can easily produce examples of $C^{(n)}$-a.p. functions. For example, let $h(x)=\exp \{f(x)\}$, where $f(x)=\sin (\alpha x)+\sin (\beta x), x \in \mathbb{R}$ and $\alpha, \beta$ are incommensurate real numbers.

EXAMPLE 4.5. It is also easy to give examples of $C^{(n)}$-a.p. functions which are not $C^{(n+1)}$-a.p. ones. For example, let

$$
f(x)= \begin{cases}\left(x-\frac{2 k}{\pi}\right)^{2} \sin \frac{1}{x-2 k / \pi} & \text { if } x \in\left[\frac{2 k-2}{\pi}, \frac{2 k+1}{\pi}\right] \backslash\left\{\frac{2 k}{\pi}\right\}, \\ 0 & \text { if } x=\frac{2 k}{\pi},\end{cases}
$$

where $k=0, \pm 1, \pm 2, \ldots$ One can easily verify that $f$ is periodic with a period $T_{f}=2 / \pi$ and $f$ is continuous on $\mathbb{R}$. Thus, $f \in A P(E)$. Now, we observe that $f^{\prime}(x)$ exists at each $x \in \mathbb{R}$ and $f^{\prime}$ is not continuous at $x=2 k / \pi$, where $k=0, \pm 1, \pm 2, \ldots$ (cf. [5]). Thus, $f$ does not belong to $A P^{(1)}(\mathbb{R})$.

The function $f$ can be utilized to construct an example for arbitrary $n \geq 2$ (see [1]).

Now, we will deal with functions of two variables $f(t, x)$. First, recall the following definition from [4] in a Banach space setting.

DEFINITION 4.6. A continuous function $f: \mathbb{R} \times E \rightarrow E$ is said to be almost periodic in $t$ for each $x \in E$ if for each $\varepsilon>0$ there exists $l>0$ such that every interval $[a, a+l]$ contains at least one point $s$ such that

$$
\|f(t+s, x)-f(t, x)\|<\varepsilon, \quad \text { for each } t \in \mathbb{R} \text { and for each } x \in E \text {. }
$$

In view of Bochner's criterion (Theorem 2.6), it is clear that the above definition is equivalent to the following one.

DEFINITION 4.7. A continuous function $f: \mathbb{R} \times E \rightarrow E$ is almost periodic in $t$ for each $x \in E$ if for every sequence of real numbers $\left(s_{n}^{\prime}\right)$, there exists a subsequence $\left(s_{n}\right)$ such that $\left(f\left(t+s_{n}, x\right)\right)$ converges uniformly in $t \in \mathbb{R}$ and $x \in E$. 
Based on Theorem 2.5, we can introduce the following definition.

Definition 4.8. A function $f: \mathbb{R} \times E \rightarrow E,(t, x) \rightarrow f(t, x)$, is said to be $(n)$-almost periodic in $t$ for each $x \in E$ if $\left(\partial^{i} f / \partial t^{i}\right)(t, x)$ is almost periodic for $i=0,1, \ldots, n$ (apparently $\partial^{0} f / \partial t^{0}=f$ ).

An analogue of Theorem 2.7 is the following one.

THEOREM 4.9. Let $f: \mathbb{R} \times E \rightarrow E$ be such that $\left(\partial^{i} f / \partial t^{i}\right)(t, x)$ are continuous for $i=$ $0,1, \ldots, n$. Then, $f$ is ( $n)$-almost periodic if and only if for every sequence of real numbers $\left(s_{n}^{\prime}\right)$, there exists a subsequence $\left(s_{n}\right)$ such that $\left(D_{1}^{(i)} f\left(t+s_{n}, x\right)\right)$ converges uniformly in $t \in \mathbb{R}$ and $x \in E, i=0,1, \ldots, n$, where $D_{1}^{(i)} f$ denotes the ith partial derivative of $f$ in view of the first variable.

REMARK 4.10. We consider the nonautonomous composition operator acting in the space $A P^{(n)}(E)$. First, we recall the corresponding result for $A P(E)$ (see [4, Lemma 3.8] in Fréchet space setting).

Let $f: \mathbb{R} \times E \rightarrow E$ be almost periodic in $t$ for each $x \in \mathbb{R}$ and assume that $f$ satisfies a Lipschitz condition in $x$ uniformly in $t \in \mathbb{R}$, that is, $\|f(t, x)-f(t, y)\| \leq L\|x-y\|$ for all $t \in \mathbb{R}$ and $x, y \in E$. Let $\varphi: \mathbb{R} \rightarrow E$ be almost periodic. Then, the function $F: \mathbb{R} \rightarrow E$ defined by $F(\varphi)(t)=f(t, \varphi(t))$ is almost periodic.

We notice that the assumptions of the above result imply that $F$ satisfies a (global) Lipschitz condition as a superposition operator acting in the space $C(\mathbb{R}, E)$.

Recall that in the case of the space $C^{(n)}(\mathbb{R}, E)$ the superposition operator $F$ satisfies the global Lipschitz condition $\left\|F\left(x_{1}\right)-F\left(x_{2}\right)\right\|_{n} \leq k\left\|x_{1}-x_{2}\right\|_{n}\left(k>0, x_{1}, x_{2} \in\right.$ $\left.C^{(n)}(\mathbb{R}, E)\right)$ if and only if the function $f$ has the form $f(s, u)=a(s)+b(s) u$ for some $a, b \in C^{(n)}(\mathbb{R}, E)$ (see [3, Theorem 8.4, page 212] or [6]). If we assume additionally that $f: \mathbb{R} \times E \rightarrow E$ is $(n)$-almost periodic in $t$ for each $x \in E$, then it is equivalent to assume that $a, b \in A P^{(n)}(\mathbb{R})$. In this situation, $F$ acts in $A P^{(n)}(E)$, obviously.

\section{Asymptotically $C^{(n)}$-almost periodic functions}

DeFINITION 5.1. A function $f \in C^{(n)}\left(\mathbb{R}_{+}, E\right)$ is said to be asymptotically $C^{(n)}$-almost periodic if it admits a decomposition

$$
f(t)=g(t)+h(t), \quad t \in \mathbb{R}
$$

where $g: \mathbb{R} \rightarrow E$ is $C^{(n)}$-almost periodic function and $h \in C^{(n)}\left(\mathbb{R}_{+}, E\right)$ with $\lim _{t \rightarrow+\infty} h(t)$ $=0$. Thus, $g$ and $h$ are called, respectively, the principal and corrective terms of the function $f$.

As an obvious consequence of the above definition and Proposition 2.10, we have the following proposition.

Proposition 5.2. Let $f_{1}, f_{2}, f: \mathbb{R}_{+} \rightarrow E, v: \mathbb{R}_{+} \rightarrow R$ be asymptotically $C^{(n)}$-almost periodic. Then, $f_{1}+f_{2}, \lambda f$ for any $\lambda \in R$ and $\nu f$ are also asymptotically $C^{(n)}$-almost periodic. 
Now, we prove the following important theorem.

THEOREM 5.3. The decomposition of a $C^{(n)}$-a.p. function is unique.

Proof. The idea of the proof is similar to that from the proof of [7, Theorem 2.5.4]. Let $f: \mathbb{R}_{+} \rightarrow E$ be $C^{(n)}$-almost periodic with two decompositions:

$$
f(t)=g_{i}(t)+h_{i}(t), \quad t \in \mathbb{R}_{+}, i=1,2,
$$

with principal terms $g_{1}, g_{2}$ and corrective terms $h_{1}, h_{2}$, respectively. Then, for $t \in \mathbb{R}_{+}$ we have

$$
g_{1}(t)-g_{2}(t)+h_{1}(t)-h_{2}(t)=0,
$$

so in view of Definition 5.1,

$$
\lim _{t \rightarrow+\infty}\left(g_{1}(t)-g_{2}(t)\right)=0
$$

Consider the sequence $(n)$. Since $g_{1}-g_{2}$ is $C^{(n)}$-almost periodic, in view of Theorem 2.7 we can extract a subsequence $\left(n_{k}\right)$ of $(n)$ such that

$$
\lim _{k \rightarrow \infty}\left(g_{1}\left(t+n_{k}\right)-g_{2}\left(t+n_{k}\right)\right)=F(t)
$$

uniformly in $t \in \mathbb{R}$. Thus, by (5.4), $F(t)=0$ for $t \in \mathbb{R}$. Since

$$
0=\lim _{k \rightarrow \infty} F\left(t-n_{k}\right)=g_{1}(t)-g_{2}(t)
$$

$h_{1}(t)=h_{2}(t)=0$ for $t \in \mathbb{R}$. The proof is complete.

In view of Proposition 5.2, we can consider the set of all $C^{(n)}$-a.p. functions $\mathbb{R}_{+} \rightarrow E$ as a linear space. We will denote it by $A_{A} P^{(n)}(E)$. Moreover, by Theorem 5.3 , the formula

$$
\|f\|_{A^{n}}=\|g\|_{n}+\|h\|_{n},
$$

where $g$ and $h$ are the principal and corrective terms of the function $f$, respectively, defines the norm on the space $A A P^{(n)}(E)$ (obviously in the case of the norm of the corrective term $h$, the supremum is taken over $\mathbb{R}_{+}$). We have the following theorem.

THEOREM 5.4. $A A P^{(n)}(E)$ considered with the norm (5.7) is a Banach space.

Proof. Let $\left(f_{n}\right)$ be a Cauchy sequence in $A A P^{(n)}(E)$. Then, the principal terms of the functions $f_{n}:\left(f_{n}\right)_{g}$ form a Cauchy sequence of $C^{(n)}$-almost periodic functions with 
respect to the norm of the space $A P^{(n)}(E)$. Thus, by Corollary 2.8, there exists a $C^{(n)}$ almost periodic function $g$ such that $\left\|\left(f_{n}\right)_{g}-g\right\|_{n} \rightarrow 0$, as $n \rightarrow \infty$. Further, the corrective terms of the functions $f_{n}:\left(f_{n}\right)_{h}$ form a Cauchy sequence in the space $C^{(n)}\left(\mathbb{R}_{+}, E\right)$. Hence, there exists a continuous function $h \in C^{(n)}\left(\mathbb{R}_{+}, E\right)$ such that $\left\|\left(f_{n}\right)_{h}-h\right\|_{n} \rightarrow 0$, as $n \rightarrow \infty$. Since for every $n \in \mathbb{N} \lim _{t \rightarrow+\infty}\left(f_{n}\right)_{h}(t)=0$ and $h(t)=h(t)-\left(f_{n}\right)_{h}(t)+\left(f_{n}\right)_{h}(t)$ for $t \in \mathbb{R}_{+}, \lim _{t \rightarrow+\infty} h(t)=0$. Thus, $f:=g+h \in A A P^{(n)}(E)$ and $\lim _{n \rightarrow \infty}\left\|f_{n}-f\right\|_{A^{n}}=0$, so $A A P^{(n)}(E)$ is a Banach space.

THEOREM 5.5. Let $E_{1}, E_{2}$ be Banach spaces and let $f: \mathbb{R}_{+} \rightarrow E_{1}$ be an asymptotically $C^{(n)}$-almost periodic function. Let $\phi: E_{1} \rightarrow E_{2}$ be a mapping of the class $C^{(n)}$. Moreover, assume that there exists a compact set $B$ which contains the closure of the set $\{h(t)$ : $\left.t \in \mathbb{R}_{+}\right\}$, where $h$ is the corrective term of $f$. Then, the function $\phi(f(t)): \mathbb{R}_{+} \rightarrow E_{2}$ is $C^{(n)}$-a.p.

Proof. Let $f(t)=g(t)+h(t)$ for $t \in \mathbb{R}_{+}$, where $g$ and $h$ are principal and corrective terms of $f$, respectively. In view of Theorem 4.3 , the function $\phi(g(t))$ is $C^{(n)}$-almost periodic. Set $\Gamma(t)=\phi(f(t))-\phi(g(t))$ for $t \in \mathbb{R}_{+}$. Obviously, $\Gamma$ is a $C^{(n)}$-mapping. Let $\varepsilon>0$. By assumption, there exists a compact set $C$ which contains the closures of $\left\{f(t): t \in \mathbb{R}_{+}\right\}$and $\left\{g(t): t \in \mathbb{R}_{+}\right\}$, so $\phi$ restricted to $C$ is uniformly continuous. Thus, there exists $\delta=\delta(\varepsilon)>0$ such that

$$
\|\phi(x)-\phi(y)\|_{2}<\varepsilon \quad \text { if }\|x-y\|_{1}<\delta, x, y \in E_{1},
$$

where $\|\cdot\|_{1},\|\cdot\|_{2}$ denote the norms of $E_{1}$ and $E_{2}$, respectively. Since $\lim _{t \rightarrow+\infty} h(t)=0$, there exists $t_{0}>0$ such that

$$
\|f(t)-g(t)\|_{1}=\|h(t)\|_{1}<\delta \text { for } t>t_{0} .
$$

Thus, we obtain

$$
\|\Gamma(t)\|_{2}=\|\phi(f(t))-\phi(g(t))\|_{2}<\varepsilon \text { for } t>t_{0} .
$$

Hence, the function $\phi(f(t))=\phi(g(t))+\Gamma(t)$ is asymptotically $C^{(n)}$-a.p.

The last result of this section concerns the integration of asymptotically $C^{(n)}$-almost periodic functions.

THEOREM 5.6. Let $E$ be a Banach space and $f: \mathbb{R}_{+} \rightarrow E$ an asymptotically $C^{(n)}$-almost periodic function. Consider the function $F: \mathbb{R}_{+} \rightarrow E$ defined by $F(t)=\int_{0}^{t} f(s) d s$ and $G: \mathbb{R} \rightarrow E$ defined by $G(t)=\int_{0}^{t} g(s) d s$, where $g$ is the principal term of $f$. Assume $G$ has a relatively compact range in $E$ and that $\int_{0}^{+\infty}\|h(t)\| d t<+\infty$, where $h$ is the corrective term of $f$. Then, $F$ is asymptotically $C^{(n)}$-almost periodic; its principal term is $G(t)+\int_{0}^{+\infty} h(s) d s$ and its corrective term is $H(t)=-\int_{t}^{+\infty} h(s) d s$.

Proof. In view of Theorem 3.4, $G(t)$ is $C^{(n)}$-almost periodic. Since $\int_{0}^{+\infty} h(s) d s$ exists, $G(t)+\int_{0}^{+\infty} h(s) d s$ is almost periodic. Moreover, the function $H$ is $C^{(n)}$-mapping 
and $\lim _{t \rightarrow+\infty} H(t)=0$. Since

$$
F(t)=G(t)+\int_{0}^{+\infty} h(s) d s+H(t) \quad \text { for } t \in \mathbb{R}_{+} .
$$

The proof is complete.

ACKNOWLEDGMENT. We would like to thank Dr. Toka Diagana for all his comments.

\section{REFERENCES}

[1] M. Adamczak, $C^{(n)}$-almost periodic functions, Comment. Math. Prace Mat. 37 (1997), 1-12.

[2] M. Adamczak and S. Stoínski, On the $\left(N C^{(n)}\right)$-almost periodic functions, Proceedings of the 6th Conference Function Spaces (R. Grzáslewicz, Cz. Ryll-Nardzewski, H. Hudzik, and J. Musielak, eds.), World Scientific Publishing, New Jersey, 2003, pp. 39-48.

[3] J. Appell and P. P. Zabrejko, Nonlinear Superposition Operators, Cambridge Tracts in Mathematics, vol. 95, Cambridge University Press, Cambridge, 1990.

[4] D. Bugajewski and G. M. N'Guérékata, Almost periodicity in Fréchet spaces, J. Math. Anal. Appl. 299 (2004), no. 2, 534-549.

[5] H. and J. Musielakowie, Mathematical Analysis, Vol. I, WN UAM, Poznań, 1993.

[6] J. Matkowski, Form of Lipschitz operators of substitution in Banach spaces of differentiable functions, Zeszyty Nauk. Politech. Łódz. Mat. (1984), no. 17, 5-10.

[7] G. M. N'Guérékata, Almost Automorphic and Almost Periodic Functions in Abstract Spaces, Kluwer Academic/Plenum Publishers, New York, 2001.

Dariusz Bugajewski: Faculty of Mathematics and Computer Science, Adam Mickiewicz University, Umultowska 87, Poznań 61-614, Poland

E-mail address: ddbb@amu .edu.p1

Gaston M. N'Guérékata: Department of Mathematics, Morgan State University, 1700 E. Cold Spring Lane, Baltimore, MD 21251, USA

E-mail address: gnguerek@morgan.edu 


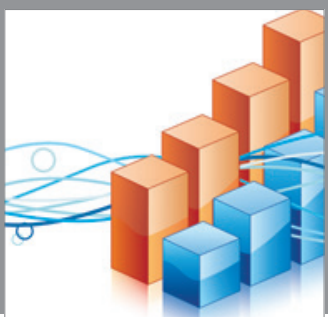

Advances in

Operations Research

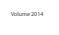

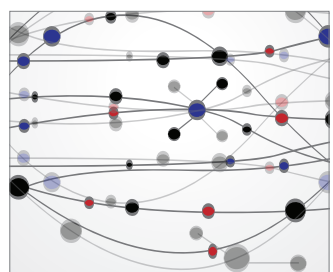

\section{The Scientific} World Journal
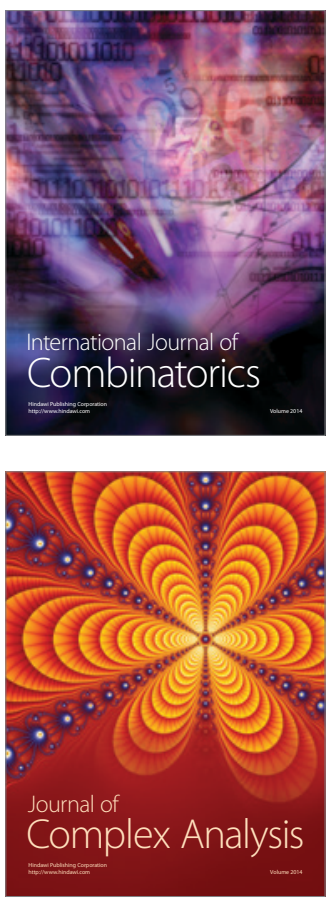

International Journal of

Mathematics and

Mathematical

Sciences
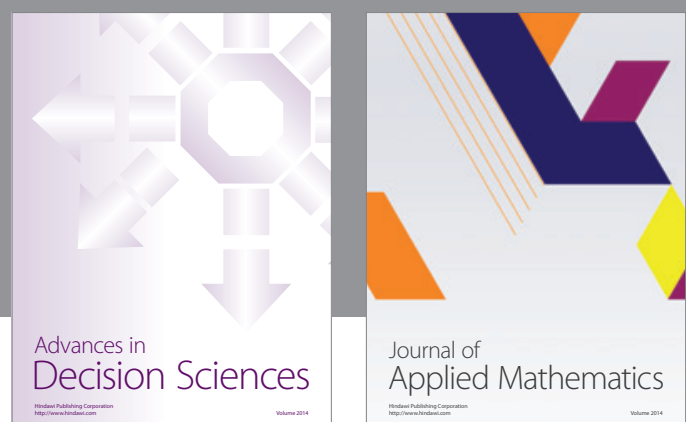

Journal of

Applied Mathematics
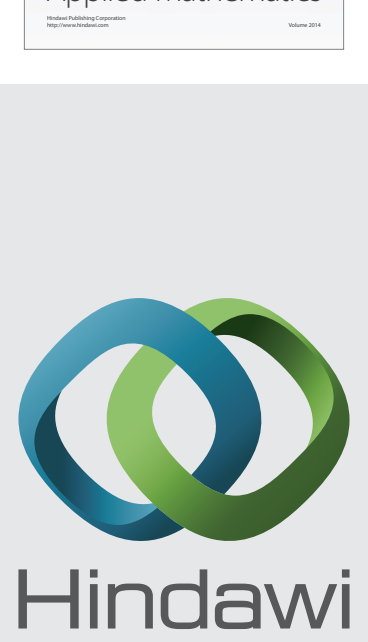

Submit your manuscripts at http://www.hindawi.com
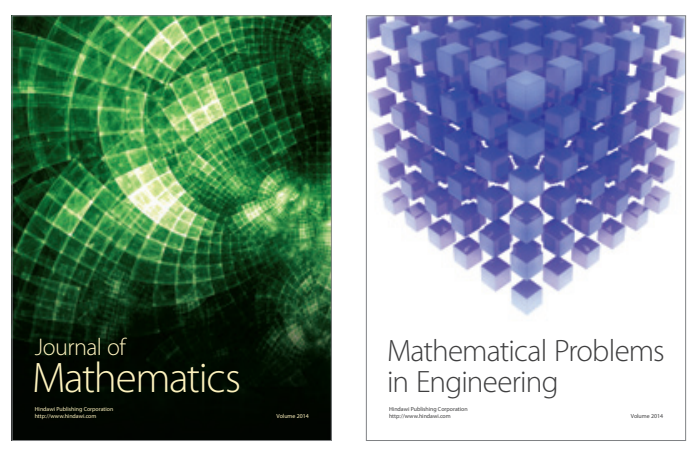

Mathematical Problems in Engineering
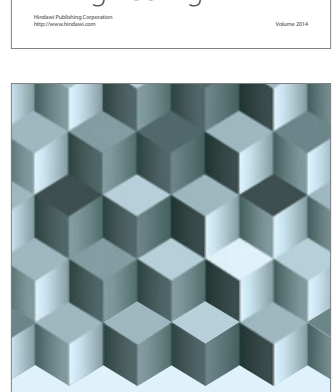

Journal of

Function Spaces
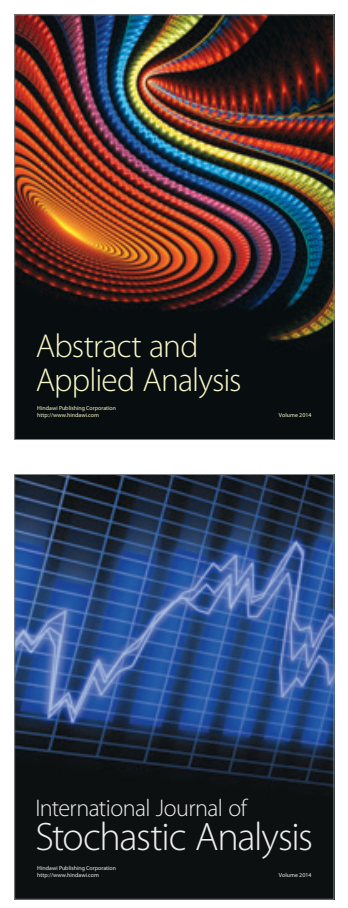

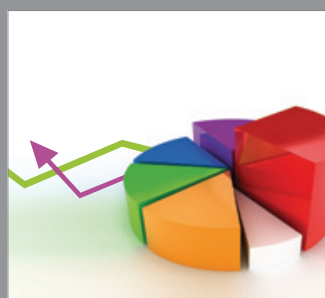

ournal of

Probability and Statistics

Promensencen
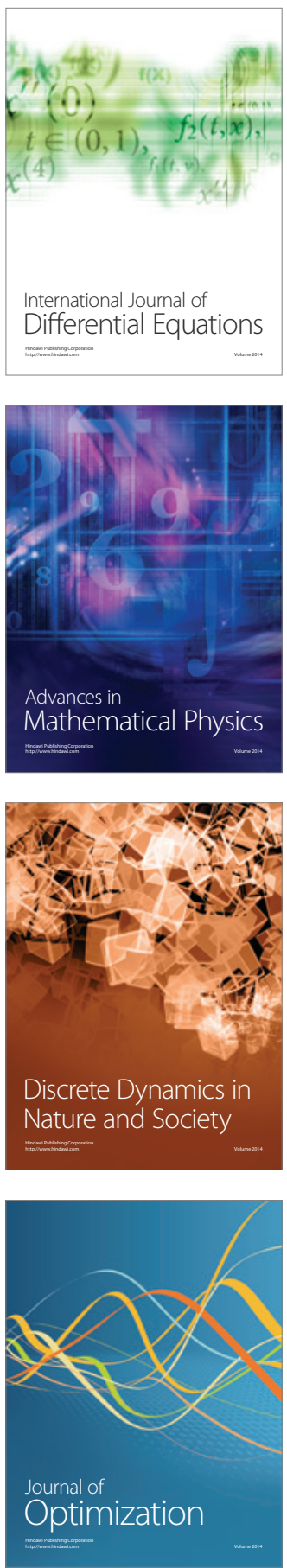